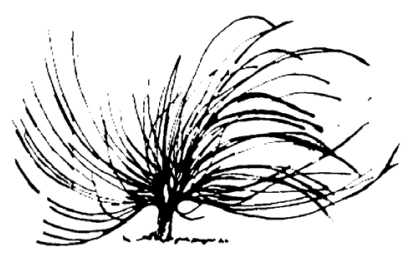

\title{
Habitar un territorio transfronterizo: propuesta educativa no formal dirigida a jóvenes rurales del cantón de Upala, Alajuela, Costa Rica
}

\author{
Erick Francisco Salas-Acuña ${ }^{l}$ \\ Tecnológico de Costa Rica \\ Costa Rica \\ esalas@itcr.ac.cr
}

\begin{abstract}
Oriana Sujey Ortiz-Vindas ${ }^{2}$
Consultora independiente

Costa Rica

oriana.ortiz.vindas@gmail.com
\end{abstract}
Uriel Antonio Trejos-Trejos ${ }^{3}$
Ministerio de Educación Pública
Costa Rica
uritrejos@hotmail.com

\begin{abstract}
Resumen
Este trabajo describe una propuesta de educación no formal desarrollada en el cantón transfronterizo de Upala, Alajuela, Costa Rica. El proceso educativo se llevó a cabo durante el 2017, con la participación de jóvenes pertenecientes a la Red de Jóvenes Migrantes y Transfronterizos, quienes se incorporaron a una serie de talleres dirigidos a la reflexión en torno a lo que
\end{abstract}

\section{$(\mathbb{\theta} \Theta \Theta$}

Recibido: 18 de julio de 2019. Aprobado: 28 de octubre de 2019.

http://dx.doi.org/10.15359/rep.15-1.9

1 Magíster en Educación Rural Centroamericana, Universidad Nacional, Heredia, Costa Rica.

2 Magíster en Educación Rural Centroamericana, Universidad Nacional, Heredia, Costa Rica.

3 Magíster en Educación Rural Centroamericana, Universidad Nacional, Heredia, Costa Rica. 
significa habitar un territorio rural transfronterizo. Se parte del hecho de que los jóvenes constituyen una población marcada por una exclusión estructural fundada en nociones adultocéntricas que, en el caso particular de esta zona, cobra aún mayor dimensión debido a la condición de ruralidad y migración que históricamente ha caracterizado a estas poblaciones. Upala, cantón rural transfronterizo en la región norte del país, constituye un territorio cuyas dinámicas particulares reflejan una realidad social compleja, pocas veces atendida por las políticas económicas, culturales y educativas de los gobiernos de turno. Este proyecto constituye un esfuerzo por atender a esta población vulnerable, con el fin de reflexionar en torno a su condición de jóvenes rurales migrantes. Se argumenta a favor de la necesidad de promover la inclusión de estos grupos, mediante iniciativas gubernamentales más acordes con las condiciones propias de estas regiones.

Palabras clave: educación rural, educación no formal, jóvenes, nueva ruralidad, zona trasfronteriza, interculturalidad.

\begin{abstract}
This paper describes a non-formal education proposal developed in the cross-border canton of Upala, Alajuela, Costa Rica. The educational process was carried out during 2017 with the participation of young people belonging to the Network of Young Immigrants and Transborder, who participated in a series of workshops aimed at reflecting on what it means to inhabit a transboundary rural territory. It starts from the fact that young people constitute a population marked by a structural exclusion based on adult-centered notions that, in the particular case of this area, take on even greater dimensions due to the condition of rurality and immigration that has historically characterized these populations. Upala, a trans-border rural canton in the northern region of the country, constitutes a territory whose particular dynamics reflect a complex social reality rarely addressed by the economic, cultural and educational policies of the governments of the day. This
\end{abstract}


Habitar un territorio transfronterizo: propuesta educativa no formal dirigida a jóvenes rurales del cantón de Upala, Alajuela, Costa Rica

project is an effort to serve this vulnerable population in order to reflect on their status as young rural migrants. It is argued in favor of the need to promote the inclusion of these groups through governmental initiatives more in keeping with the conditions of these regions.

Keywords: rural education, non-formal education, youth, new rurality, cross-border area, interculturality.

\section{Introducción}

$\mathrm{E}$

n la actualidad, las comunidades rurales enfrentan un debilitamiento progresivo de sus identidades, debido a diversos procesos, entre ellos la ampliación de sus vías de comunicación, la migración, la influencia de los medios de comunicación masiva, la economía transnacional, entre otros. Como consecuencia, los modos de vida asociados al campo resultan cada vez más amenazados, al ceder ante las nuevas demandas que imponen estos cambios sobre las actividades económicas tradicionales. De ahí que procurar espacios novedosos de reflexión para la reconstrucción de nuevas identidades rurales sea fundamental, si lo que se quiere es promover un desarrollo más acorde con las condiciones y necesidades de estas comunidades. Lo anterior, sobre todo, porque la identidad está en constante construcción y demanda, por ende, cada vez más, de nuevos procesos que orienten su comprensión.

No obstante, el problema con las comunidades rurales, por lo menos en el caso costarricense, no solo ha consistido en que han sido pocos los esfuerzos por plantear políticas según las diferencias culturales, económicas, geográficas y étnicas de estos pueblos, sino que, cuando estas han existido, se basan en visiones estáticas acerca de lo rural, que obvian las transformaciones acaecidas a lo largo de los últimos años, reproduciendo, de esta forma, discursos que no corresponden con la realidad (Angulo, Morera y Torres, 2010). Es decir, existe un desfase entre el discurso (la teoría) y la realidad (la práctica) que no solo afecta la comprensión de lo rural, sino que además limita la implementación de políticas públicas que contribuyan al mejoramiento de la vida de estas poblaciones. El reto, por tanto, empieza por hacer que estos discursos sobre lo rural se actualicen, para que logren adaptarse a las condiciones particulares que presentan estos territorios. Esta transformación, sin embargo, no solo implica que los gobiernos reformulen 
sus políticas con el afán de hacerlas más inclusivas, sino también que las comunidades rurales logren reconocerse como parte de un territorio $\mathrm{y}$, en este sentido, logren valorizar tanto su vida como sus costumbres en medio del nuevo contexto político económico.

Dentro de este escenario, la educación constituye una herramienta fundamental para la reconstrucción de procesos identitarios que contribuyan a la revalorización de lo rural desde supuestos multiculturales y pluriétnicos. Para esto, es necesario que las políticas educativas se actualicen desde un enfoque territorial que responda a las particularidades sociales, económicas y culturales de las zonas rurales, de manera que quienes asistan a la educación primaria y secundaria puedan asumirse como herederos de una tradición que merece ser valorada, conservada e incluida en el imaginario nacional.

En este sentido, este trabajo procura convertirse en un esfuerzo por desarrollar un procedimiento educativo no formal con jóvenes de un territorio rural, el cantón de Upala, San Carlos, Alajuela, Costa Rica, con el fin de posicionar la discusión sobre su identidad, que parta de la comprensión de las transformaciones identitarias producidas a raíz de los cambios en los modos de vida, producto de los nuevos sistemas de producción y los procesos de migración de los últimos años. Se espera, como consecuencia de esta reflexión, contribuir al empoderamiento de actores, en este caso, líderes jóvenes, quienes, a partir del reconocimiento de las nuevas condiciones que caracterizan actualmente su ruralidad, puedan profundizar en el cuestionamiento acerca del sentido de frontera desde lo territorial y simbólico.

Al preguntarse acerca de lo que significa ser y estar en su territorio, se cree que estos jóvenes puedan establecer mayores vínculos favorecedores de procesos de integración social. Al fortalecer el involucramiento de la juventud y su participación activa en la toma de decisiones en el nivel local, se espera construir mejores relaciones de convivencia en un espacio fronterizo, basadas en la valoración de las diferencias culturales que contiene su territorio. Se trata, en esencia, de que el tema de la identidad se construya desde lo rural, a través de la valorización del potencial geográfico, cultural, étnico e histórico, como parte fundamental de la riqueza de los pueblos.

En el cantón de Upala, converge una gran cantidad de problemáticas que contribuyen a que sea una de las zonas con menores índices de desarrollo en el nivel nacional (MIDEPLAN, 2013). Por ejemplo, 
existen pocas opciones de empleabilidad, los sistemas de producción obedecen cada vez a dinámicas latifundistas que se alejan de la otrora agricultura tradicional, lo que causa que la tenencia de las tierras por los campesinos pase a manos de las grandes empresas (Programa Conjunto Juventud, Empleo y Migración, 2011). Asimismo, los modos de vida asociados al campo pierden valor ante la influencia de estilos de vida globalizados. Frente a esto, sin embargo, los gobiernos locales poco hacen para proponer políticas de desarrollo acordes con las necesidades y problemas de esta comunidad, lo que contribuye a incrementar aún más su vulnerabilidad. A este panorama se le une la poca organización local que, por una parte, carece de las herramientas para comprender el devenir de su situación tanto social como política y, por otra, la falta de liderazgos que propongan soluciones basadas en una identidad revalorizadora de lo rural, con el fin de construir vínculos comunitarios que se proyecten a futuro. Asimismo, la comunidad está influenciada por los procesos migratorios, debido a su cercanía con Nicaragua, condición que, aunque ya es de larga data, no se posiciona como un elemento que forme parte de un proyecto identitario reconocedor del carácter binacional, el cual englobe las dinámicas sociales que allí toman lugar (Rodríguez, 2001; León-Araya, 2015; Delgado y Córdoba, 2012).

Abordar estas problemáticas constituye, por lo tanto, un aporte para los estudios en torno a la identidad, que, por lo general, se enfocan desde perspectivas nacionales, obviando las diferencias regionales que pueden estar presentes. Asimismo, implica reconocer que la identidad es algo en constante construcción, que, por tanto, requiere nuevas aproximaciones que estudien los procesos económicos, políticos y culturales, los cuales influyen en los modos de ser de las personas para lograr sociedades más justas e inclusivas.

La necesidad de construir procesos orientados a resolver las problemáticas que viven los jóvenes del cantón de Upala pasa obligatoriamente por la resignificación de lo que implica ser joven en este contexto. En este sentido, y a la luz de los cambios que experimenta este cantón, parece necesario que las personas jóvenes puedan contar con espacios que les permitan reconocerse como integrantes de una comunidad con condiciones particulares. Solo así es posible que ellos puedan comprender mejor el lugar donde viven y asumirse como agentes de cambio de la comunidad, con el objeto de luchar por construir mejores alternativas a futuro para la población joven del cantón. En este sentido, 
la pregunta que interesa responder en este trabajo es la siguiente: ¿Qué significa ser joven en las nuevas condiciones sociales, económicas y culturales que enfrenta el cantón de Upala?

El aporte metodológico propuesto, basado en una aproximación desde la educación rural no formal, ofrece también una ventaja, en tanto se perfila como una acción complementaria a la educación formal. Esto favorece mucha más flexibilidad y libertad para educar a las personas de las comunidades rurales acerca de cómo revalorizar la riqueza de lo rural, no solo para una mejor comprensión de sí mismas, sino también para proyectarse hacia el futuro. Pensar la identidad no únicamente desde lo nacional, sino también desde los distintos espacios regionales, en este caso a través de propuestas educativas no formales, puede contribuir a la conformación de un imaginario más diverso desde el punto de vista intercultural, que eventualmente pueda materializarse mediante políticas más inclusivas y respetuosas de las diferencias.

\section{Los jóvenes como nuevos sujetos históricos}

Trabajar con poblaciones jóvenes pasa necesariamente por repensar los discursos que en general han construido las instituciones sociales (la Iglesia, la educación, la familia, los partidos políticos, el mercado y las ciencias sociales) en torno a esta población. Esto porque el habla social que se reproduce sobre los jóvenes desde estas instancias tiende, habitualmente, a asumir la juventud desde nociones homogeneizantes que poco contribuyen a la comprensión de este grupo social. Por ejemplo, la mayor parte de trabajos sobre esta población, como señala Duarte (2006), se olvidan de que no solo es esencial distinguir entre jóvenes que habitan en zonas rurales o urbanas, sino que, además, resulta obligatorio considerar las especificidades en relación con su raza, etnia, género o clase social. "La juventud, para muchos investigadores e investigadoras de nuestra época, es una condición natural sin diferencias, definida por su proceso psicobiológico, independientemente de los condicionamientos históricos, económicos y culturales que la producen" (Duarte, 2006, p. 30).

Según ese autor, el resultado de estas aproximaciones ha derivado en la reproducción de un discurso en torno a lo que la sociedad entiende por "la juventud", marcado por tres características: 1) un predominio de aproximaciones realizadas en su mayoría por adultos, que excluyen las contribuciones de los jóvenes en la construcción de prácticas 
y significados sobre su edad; 2) investigaciones y trabajos que, al no cuestionar este discurso, terminan por reafirmar ideas en torno a él, basadas en un adultocentrismo que restringe la definición de ser joven a un momento de preparación para la vida adulta, y, por último, 3) la universalización del estereotipo de "rebeldía juvenil" como un disvalor que atenta contra el orden social y su integración en la sociedad.

Estas ideas reproducidas en muchas de las investigaciones y trabajos que realizados con poblaciones jóvenes constituyen hoy en día el mayor obstáculo por vencer, si lo que se quiere es buscar la posibilidad de construir nuevas miradas que permitan conocer a los jóvenes desde lo que Zúñiga (2010) denomina una "práctica histórica de la edad", es decir, como vivencia histórica de un periodo vital. Esto pasa no solo por romper con el discurso adultocéntrico que define la identidad del joven en relación con el adulto, sino, sobre todo, con reconocer la juventud como una etapa que tiene su propia identidad y en la cual los mismos jóvenes tienen en derecho a hablar y a pensarse desde sus experiencias, inquietudes y sueños (Duarte, 2006).

El trabajo de Zúñiga (2010) reconoce que la investigación sobre los jóvenes en América Latina y el Caribe en la actualidad está marcada por tres tendencias esenciales. Por un lado, se encuentra la vertiente psicologista, para la cual ser joven significa estar en una etapa de preparación para la edad adulta; es decir, se es joven por negación y subordinación a la experiencia y madurez que caracteriza la vida adulta. De ahí que se conciba al joven como un no adulto, alguien cuya identidad aún se encuentra en conflicto, disgregada, no definida, hasta el momento en que finalmente asuma una única e inamovible. Se trata de una especie de moratoria social en la cual se desvaloriza la juventud, al encontrarse en una etapa de transición en la que se concibe la persona como adulta incompleta que deben ensayar diferentes identidades hasta asumir las actitudes y normas que caracterizan la vida adulta.

Por otra parte, están los estudios cuantitativistas, para los que los jóvenes son capital humano, fuerza de trabajo, potencial productivo, etc. Se trata de aproximaciones en las que los jóvenes existen en cuanto potencial de riqueza y capital, calculados en tasas, porcentajes e índices, pero no como creadores de cultura (Zúñiga, 2010). Esta es la visión reproducida desde muchas de las organizaciones que ven a los jóvenes únicamente desde criterios demográficos, privilegiando lo cuantitativo por sobre lo cualitativo. "No ven personas ni sujetos con capacidades 
y potencias liberadoras, no ven presente (nada más futuro), no ven sentimientos legítimos, solo problemas y recambio para asegurar el funcionamiento de su sistema de vida (y de muerte)" (Duarte, 2006, p. 31). El resultado de reducir el concepto de juventud a puro conocimiento "técnico" es la creencia de que la solución radica en aplicarles políticas públicas, ignorando el trasfondo de los problemas sociales y, por ende, sacrificando los análisis en profundidad sobre las meras descripciones.

Como un intento por superar las dos tendencias anteriores, se encuentran las aproximaciones que buscan asumir a las personas jóvenes como sujetos. Para estos trabajos, comprender la juventud implica entender las condiciones históricas, puesto que se trata de "una categoría sujeta a las diversas condiciones, sociales y subjetivas, por las que atraviesan las sociedades y las personas" (Zúñiga, 2010, p. 74). Esta definición supone el esfuerzo por producir trabajos con poblaciones jóvenes más contextualizados y dinámicos, pues significa mirar desde la diversidad a un mundo social que, por lo general, es reducido a perspectivas unívocas y homogeneizantes invisibilizadoras de la pluralidad y el aporte de este sector en las sociedades.

Se trata ya no de hablar de "juventud", sino más bien de "juventudes", para reflejar el abanico amplio de significaciones que resulta tanto de la contextualización como de las especificidades, y que promueve el reconocimiento de la heterogeneidad, la diversidad y la pluralidad de este grupo social. Duarte (2006, pp. 67-68) explica este cambio de mirada sobre las juventudes, de la siguiente manera:

Vale decir, se propone leer lo juvenil desde sus aportes y no desde sus carencias, cuestión que no pretende invalidar las dificultades sociales y de otro tipo existentes en estos mundos juveniles, nada más que enfatiza otro ámbito de la mirada, hacia las posibilidades que desde este grupo social se generan y no desde las ausencias o carencias que tienen.

Con el propósito de mirar, aprehender y comprender las juventudes desde nuevas matrices analíticas, el trabajo de Duarte (2006) propone una serie de pistas que se ofrecen como herramientas epistemológicas para un abordaje de este grupo social. La primera de sus pistas está relacionada con la necesidad de diferenciar la singularidad que representan los estilos culturales y los subgrupos etáreos en la 
construcción de los mundos juveniles. Es decir, se trata de entender que la construcción de la realidad es distinta a los 15 y a los 29 años, al igual que lo es pertenecer a un grupo de rap o a uno de rock.

La segunda pista está enlazada con la importancia de promover acercamientos desde lo juvenil, que permitan desplegar la pluralidad y riqueza del mundo juvenil. En relación con esto, Duarte (2006, p. 134) expresa lo siguiente:

Por largo tiempo, las miradas predominantes son desde la lejanía, desde el escritorio de la oficina pública, la ONG, la academia, la iglesia Se requiere en este nuevo esfuerzo epistemológico salir a la calle, vincularse con las y los jóvenes, oír sus hablas, mirar sus acciones, sentir sus aromas.

La otra pista está asociada a la vinculación de las juventudes con la comprensión de las realidades sociales, a través de la historización de las experiencias juveniles y el análisis del papel que tienen las generaciones en la construcción de una memoria colectiva.

Esta categoría relacional: lo generacional, nos permite pensar y comprender acciones, discurso, cosmovisiones, sentimientos y otras formas de vida de los grupos juveniles en distintos momentos de la historia, desde los estilos que las relaciones sociales que asumen van tomando, en directa relación con otros grupos sociales - adultos, adultos mayores, niñez - y entre ellos mismos (Duarte, 2006, p. 72).

Finalmente, una última pista propone la posibilidad de superar las categorías que han dominado hasta ahora la discusión en torno a la juventud, para dar paso a un nuevo lenguaje más apropiado al dinamismo y a la heterogeneidad del mundo juvenil, tal y como lo indica Duarte (2006, p. 137):

En este sentido, planteamos la necesaria construcción de conceptos en torno al mundo juvenil, no en la prospección de crear categorías totalizantes y universalizadoras, cuanto conceptos dinámicos y flexibles que se acerquen progresivamente a los 
sujetos de estudio: las y los jóvenes, las juventudes, las expresiones juveniles, los procesos de juvenilizacción.

De esta manera, estudios como los realizados por autores como Duarte (2006) y Zúñiga (2010) proponen la necesidad de construir nuevas categorías epistemológicas que superen las aproximaciones psicologistas y cuantitativistas, en busca de miradas novedosas reconocedoras de la juventud como una experiencia vital que merece ser aprendida desde los contextos y condiciones particulares que la definen. Duarte (2006) lo explica del siguiente modo:

Las juventudes cobran vida, se muestran, nos muestran sus diferentes estéticas y podemos asumir entonces una episteme integradora, amplia y comprensiva de lo juvenil. La juventud niega existencia porque encajona, cierra y mecaniza las miradas; rigidiza y superfializa el complejo entramado social que hemos denominado las juventudes. Vamos por el camino del reconocer diferencias, aceptar diversidades, construir aceptaciones y de esa forma construimos miradas potenciadoras de lo juvenil (p. 143).

\section{Descripción de la propuesta educativa}

El trabajo de la Red de Jóvenes Migrantes y Transfronterizos constituye un punto de partida para cualquier experiencia que pretenda construir agendas de trabajo con los jóvenes del cantón. De ahí que el primer paso para este proyecto consistió en un diagnóstico con los jóvenes integrantes de la Red, con el fin de recolectar los insumos necesarios que servirán para desarrollar el proceso educativo. Con este fin, se efectuó una revisión bibliográfica de informes y documentos construidos por la Red, como la Agenda Joven, y se procedió a la realización de entrevistas a varios de sus integrantes. La información recolectada sirvió para construir una propuesta educativa no formal que dirigida a sensibilizar a los jóvenes de la Red, en torno a su identidad en un territorio marcado por las dinámicas transfronterizas y las transformaciones de los medios tradicionales de producción basados en la agricultura campesina. La condición de ser joven migrante pone en mayor grado de vulnerabilidad a esta población, sobre todo a aquella en condición de irregularidad migratoria. 
La propuesta modular de talleres pasa por la definición de su identidad individual, familiar y colectiva, hasta llegar al contexto local, con el afán de indagar en su memoria histórica y así poder reconocerse en su dinámica intercultural, todo en función reconstruir referentes identitarios anclados en lo que significa para estos jóvenes habitar un territorio transfronterizo. El trabajo involucró tres fases: 1) un diagnóstico participativo, 2) el diseño de una propuesta de talleres y 3 ) un proceso de socialización de conocimientos. En cada una de ellas, hubo, a su vez, una serie de pasos necesarios para obtener la información que permitiera cumplir con cada uno de los objetivos propuestos en este trabajo, tal y como se presenta en la figura 1.

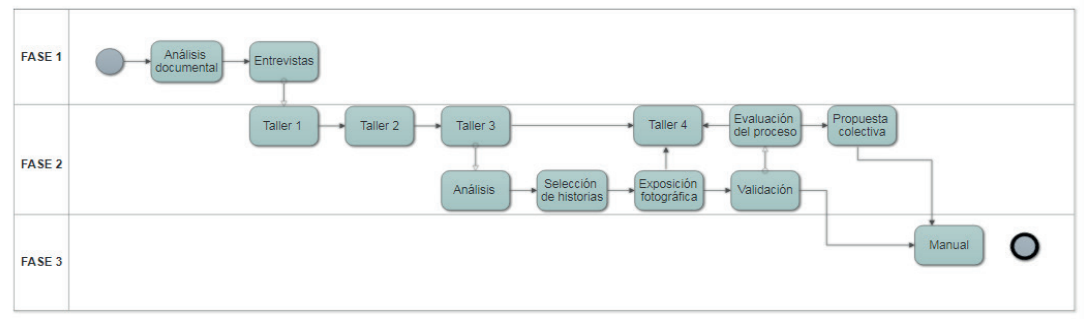

Figura 1. Diagrama de las fases de la propuesta

Nota: Elaboración propia.

En la tabla 1, se describe el contenido, las actividades y los resultados de cada uno de los talleres desarrollados. Como puede verse, la fase 1 del proceso consistió en un diagnóstico participativo del trabajo realizado por la Red, el cual incluyó la consulta de material bibliográfico y la ejecución de entrevistas a los jóvenes integrantes, con el fin de conocer, de primera mano, su percepción en torno a la experiencia de trabajo con la organización. Producto de este primer diagnóstico, se elabora una propuesta de talleres basada en las necesidades detectadas, sobre las cuales se construye un proceso orientado a la reflexión en torno a su condición de jóvenes rurales que habitan un territorio transfronterizo. En esta fase, interesa reconocer, por ejemplo, la composición del grupo, la metodología utilizada, los niveles de participación de la juventud, las causas de deserción o permanencia, los procesos formativos indirectos generados, entre otros.

La fase 2 comprendió la realización de cuatro talleres, tres de los cuales se llevaron a cabo durante abril, en Upala, y uno en setiembre, 


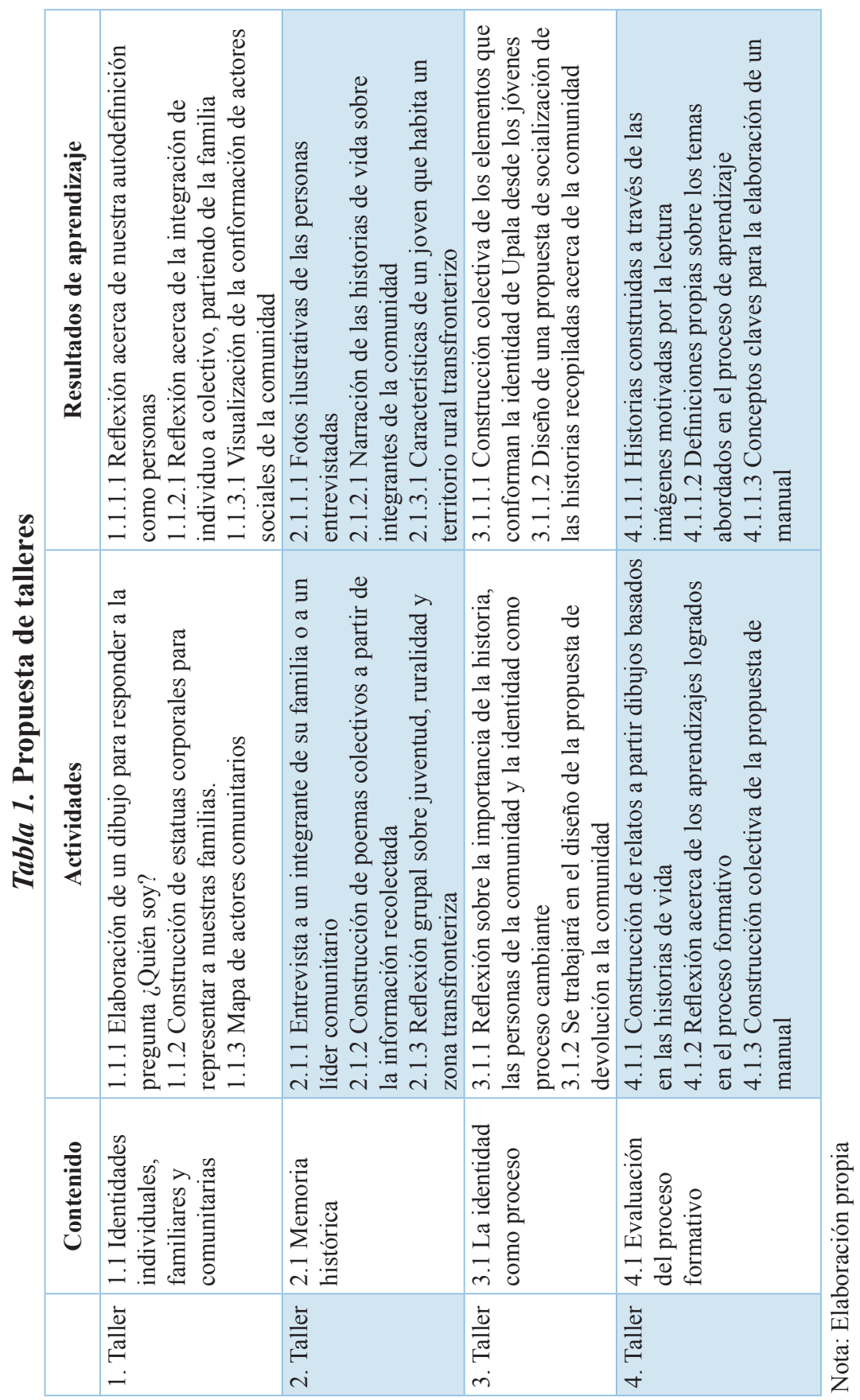


aprovechando los espacios de trabajo propios de la Red. La reflexión generada a partir de los dos primeros talleres sirvió como insumo para ejecutar un trabajo de campo, que consistió en una exposición fotográfica como una manera de proyectar lo aprendido en los talleres y de contribuir al rescate de la memoria histórica de la comunidad de Upala. El taller cuatro, finalmente, comprendió la evaluación de la experiencia, incluida una propuesta colectiva y validada del manual sugerido como el producto final de todo el proceso.

La fase 3, finalmente, procuró condensar la experiencia mediante la elaboración, edición e impresión de un manual orientado a la reflexión sobre la condición transfronteriza de la identidad de los jóvenes de Upala, que sirva como insumo a la Red para nutrir el diálogo a futuro con otros jóvenes y, con ello, promover una mejor comprensión de su contexto. Todo esto en aras de favorecer su involucramiento y participación en construir agendas comunitarias más justas e inclusivas para quienes habitan este territorio.

\section{Informe de resultados}

En este apartado, se ofrece una evaluación del trabajo realizado, con el fin de reconocer dificultades, obstáculos y aspectos que deberían tenerse en cuenta para futuras experiencias educativas con jóvenes quienes busquen fortalecer procesos de identidad rural en un contexto de frontera. Para ello, se hace un repaso de los logros alcanzados en la fase 2 , tomando en cuenta los aciertos y desaciertos, con el propósito de establecer las lecciones aprendidas. Por último, se propone un segmento de conclusiones y recomendaciones que deberán tenerse en cuenta a la hora de replicar esta propuesta educativa no formal en otros contextos.

En el primer espacio de encuentro con los jóvenes, se generaron reflexiones acerca de la individualidad, de cómo se teje en el plano familiar y, a su vez, con el ámbito comunitario, todo a través de actividades lúdicas que involucraron un dibujo y una estatua familiar, respectivamente. Estos ejercicios no solo propiciaron la construcción de un espacio de confianza y motivación, sino que, además, permitieron un primer acercamiento a los valores, aspiraciones personales, experiencias familiares y representaciones sobre la comunidad. 
Seguidamente, la construcción de un mapa comunitario sirvió para profundizar en la territorialidad de la comunidad, sus actores comunitarios y su cultura. En él, se identificaron grupos organizados y sus temas de acción, además de otras características culturales de la comunidad como sus tradiciones, leyendas, festividades, entre otros, lo que facilitó el reconocimiento de la riqueza de la comunidad de Upala por parte de los jóvenes. Entre esas características, los jóvenes destacaron las fiestas populares como la Feria del Maíz, las visitas a los ríos, los deportes como el béisbol, las comidas típicas y otras actividades dentro del territorio nicaragüense, lo que refleja, nuevamente, la dinámica transfronteriza de la comunidad.

Como conclusión de este primer taller, se les asignó entrevistar a una persona de la comunidad, con el objetivo de ampliar el mapa en la próxima sesión, a partir de la información recaudada. Se les sugirió poner énfasis en el vínculo entre historia personal y comunitaria, así como en la historia misma del desarrollo de la comunidad de Upala, todo a través de las historias recolectadas.

La segunda sesión inició con los jóvenes compartiendo las historias recopiladas, según lo asignado en el taller 1, mediante un círculo donde ellos contaban las historias de vida de las personas que entrevistaron, relacionadas, muchas veces, con la guerra civil en Nicaragua y la carencia de recursos económicos. Una vez hecho esto, y en grupos, se les dio la tarea de escribir un poema colectivo, a partir de los relatos escuchados, los cuales fueron compartidos en el círculo.

Seguidamente, partiendo de una nueva serie de preguntas, se buscó profundizar en lo que significa ser un joven rural transfronterizo. La percepción de la juventud en torno a la ruralidad está asociada a valores positivos como la solidaridad, el trabajo y la humildad de sus habitantes, la riqueza natural y cultural, así como la tranquilidad y la paz. Pero, a su vez, se mencionan aspectos negativos relacionados con la desigualdad, el abandono por parte del Estado, la pobreza, la escasez de servicios públicos, el monocultivo, la violación de derechos laborales y la falta de espacios para la participación de jóvenes en la comunidad.

Lo expresado por los jóvenes permite reconocer una visión de las personas habitantes en una zona rural, afianzada en una serie de características positivas, pero cuya existencia se ve amenazada por factores de tipo estructural que influyen en la vida de sus pobladores. También, fueron patentes algunos estereotipos comunes asociados a la ruralidad, 
como el retraso con respecto a la ciudad, la poca población, la falta de atractivos, la economía exclusivamente agrícola y ganadera, entre otros.

Entre las características propias de un joven que vive en una zona rural, se mencionan la humildad, la alegría, la sencillez, la timidez, y el valor por el trabajo. A pesar de enfrentar problemas familiares y económicos, así como en cuanto al acceso a la educación y otros derechos, los jóvenes rurales también manifiestan aspiraciones relacionadas con la posibilidad de concluir sus estudios, convertirse en profesionales para no terminar siendo mano de obra barata, obtener un trabajo remunerado o tener su propia empresa, contribuir a la economía familiar y, en general, alcanzar una mejor calidad de vida.

Por otro lado, los jóvenes reconocen el impacto del monocultivo, en este caso, de la piña, en la dinámica social de la comunidad de Upala. Si bien la producción de piña, por una parte, brinda trabajo a los lugareños, también ha significado la deforestación, la contaminación de la tierra, los mantos acuíferos y los ríos, generando un impacto en la biodiversidad de la zona.

Simultáneamente, el trabajo en las piñeras está asociado también al tema migratorio, que, en Upala, resulta un elemento fundamental para comprender la dinámica de la zona. Los jóvenes mencionan que, históricamente, la migración ha sido importante y ha tenido un papel fundamental en la construcción de la identidad local desde los primeros pobladores, cuyo intercambio comercial se hacía por vía fluvial, a través de los ríos Zapote, Frío, Guacalito y Niño. Se destaca que el territorio upaleño está conformado por un porcentaje importante de población migrante, cuya cultura constituye parte de su identidad, aunque también se menciona un sentido negativo de la migración, al enlazarla a la pobreza, delincuencia, violencia y falta de educación.

Este contexto transfronterizo le otorga particularidades a Upala asociadas al intercambio cultural, económico e ideológico entre costarricenses y nicaragüenses, que son bien reconocidas por los jóvenes. Si bien existe una convivencia pacífica y solidaria, también señalan que aún hay prejuicios hacia la población migrante nicaragüense que les responsabiliza por la saturación de los servicios públicos y de las fuentes de empleo.

Aunado a todo lo anterior, y a pesar de que existe una serie de instancias locales de participación (desde organizaciones gubernamentales hasta $\mathrm{ONG}$ ), los jóvenes manifiestan que su participación se ve 
coartada por el adultocentrismo, la falta de inclusión de las autoridades, el desinterés, el conformismo, la pobreza, la timidez y la rutina, así como por la falta de coordinación y de mayores recursos. Con todo, es notable que la juventud sí ha podido ordenarse, creando espacios por su cuenta para el involucramiento y la organización de diversas actividades, con el fin de ser tomada en cuenta.

Entre las estrategias más importantes para lograr sus aspiraciones, los jóvenes mencionaron la necesidad de crear espacios que incentiven la colectividad, a través de procesos educativos, de apoyar los proyectos comunitarios, así como de construir nuevos referentes desde la valorización de lo rural y la diversidad cultural, entre otras iniciativas orientadas propiamente a que existan mejores opciones de empleo en la zona. Por otra parte, entre los temas que los jóvenes consideran más importantes para su comunidad, se encuentran el trabajo, la educación, el medio ambiente, las drogas y la migración.

En general, cabe mencionar que, en las respuestas y discusiones, se muestra una visión ambigua basada en la oposición entre campo y ciudad, que, al tiempo que asocia lo rural al aislamiento y la carencia, por ejemplo, también reconoce el potencial natural, cultural y, sobre todo, humano de las zonas rurales. En otras palabras, si bien los jóvenes señalan algunos aspectos negativos sobre la ruralidad, estos más bien parecen ser consecuencia de una desigualdad estructural - la cual tiende a ser mayor en las zonas transfronterizas - que del territorio y de las personas que lo habitan.

La tercera sesión tuvo como objetivo principal discutir una estrategia, con la idea de compartir con la comunidad algunas de las reflexiones sostenidas como parte del proceso. Para tal efecto, se seleccionó una exposición de fotografías a gran formato, en las cuales se retratasen las historias de vida que favorezcan la reconstrucción de memoria histórica de la comunidad. Los temas seleccionados fueron: la Upala de antaño, el vínculo de la comunidad con la guerra civil nicaragüense y el papel del trabajo político que realizan los jóvenes a través de la Red. Para la exposición, se seleccionó la parada de buses vieja, por tratarse de un lugar público y concurrido.

Los jóvenes trabajaron en grupos el diseño de propuesta y gestión para devolución a la comunidad. Con este propósito, iniciaron con la realización de entrevistas a diversas personas de la misma comunidad, sobre todo, adultos mayores que formaron parte de los primeros 
pobladores de Upala. Seguidamente, se tomaron fotografías, redactaron los textos y se dispusieron a realizar la exposición en el lugar seleccionado.

Por último, la cuarta sesión inició con la lectura de la versión final de los relatos colocados en la exposición fotográfica de devolución a la comunidad. Utilizando las historias como insumo, se les solicitó a los participantes realizar un dibujo, el cual luego presentaron ante el grupo. Posteriormente, se continuó con un juego de rompecabezas, con el que, a partir de los dibujos realizados, los jóvenes procuraban reconstruir un relato coherente que integrara su trabajo y el de los demás compañeros.

La segunda parte de la sesión consistió en revisar el contenido del manual, utilizando un borrador sobre el cual los jóvenes harían sus aportes, recomendaciones y cambios. La mayoría de sugerencias tuvo que ver con el uso de un vocabulario más sencillo, además, de cambios relacionados con el avatar del manual, con el fin de ajustar su apariencia a la de una joven upaleña. Este manual constituye un esfuerzo por brindar una herramienta didáctica a la Red, que permita replicar el proceso con otros jóvenes de la comunidad. En él, se describe paso a paso una serie de actividades orientadas a que los participantes inicien un recorrido de aprendizaje, a partir de su autodefinición como individuos, para pasar al vínculo familiar y, finalmente, al tejido comunitario.

Finalmente, aprovechando el espacio de la última sesión, se aplicó un cuestionario para conocer el parecer de los jóvenes sobre el proceso realizado. El instrumento aplicado comprendía doce preguntas, las cuales buscaban evaluar aspectos tales como los temas, metodologías, materiales, entre otros, cuyos resultados fueron, en general, satisfactorios. Por ejemplo, en cuanto a la duración del proceso y al tiempo de los talleres, todos los jóvenes manifestaron que fue suficiente para el aprovechamiento máximo de las actividades, sobre todo porque siempre se buscó ajustar los horarios a las posibilidades y la disponibilidad de los participantes. Señalan, sin embargo, la inconstancia de la participación de algunos jóvenes, durante el proceso, como un problema, debido a cuestiones laborales, o bien de estudio. Con todo, destacan que el tiempo asignado fue suficiente para obtener buenos resultados y conclusiones importantes sobre lo que es vivir en una zona rural transfronteriza.

De igual modo, se expresaron en cuanto a la metodología empleada en los talleres, la cual fue calificada de creativa, accesible y propicia 
para la participación. Además, se hizo hincapié en el enfoque ludopedagógico de las actividades, que logró mantener la atención de los jóvenes en las distintas actividades. Los materiales utilizados también fueron buenos, pertinentes y acordes con cada una de las actividades. En cuanto a las dinámicas realizadas, destacan la construcción del mapa y de las historias basadas en las entrevistas, pues ambas contribuyeron a conocer más su comunidad, así como a profundizar la historia, a través de los relatos de quienes han estado involucrados en el desarrollo de Upala.

Los facilitadores, por su parte, merecieron buenas apreciaciones en general. Se señala que cumplieron con las expectativas, porque las instrucciones de las actividades fueron claras y realizadas en un espacio de paciencia, flexibilidad, amabilidad, respeto, ánimo y consideración. Unido a esto, se encuentra el hecho de que en los talleres siempre se promovió la horizontalidad y la buena comunicación, que contribuyeron a una atmósfera de confianza propicia para compartir conocimientos y experiencias.

Las expectativas de los jóvenes, por otra parte, aunque se cumplieron para la mayoría, en algunos casos se vieron limitadas, debido a la escasa participación de muchos de ellos en la Red. Expresan que se logró obtener nuevos conocimientos relacionados con la historia y la cultura, a través de la identificación de los diferentes actores socioculturales de la comunidad y de las entrevistas. También, se menciona que el espacio fue propicio para compartir, sentirse incluido e intercambiar conocimientos con los compañeros.

De igual modo, cuando se les preguntó acerca de la pertinencia de los temas abordados para la reflexión en torno a lo que significa vivir en una zona rural transfronteriza, si bien la mayoría consideró que fueron importantes, algunos manifestaron que la discusión se vio parcialmente limitada, dado que esas temáticas eran abordadas desde perspectivas muy generales. Con todo, los jóvenes manifestaron que su percepción sobre su condición había cambiado, pues el proceso les había permitido ampliar su visión sobre el lugar en donde viven. Les ayudó a deshacerse de estereotipos, profundizar en el concepto de ruralidad y entender que esta va más allá de vivir en el campo, de actividades económicas relacionadas exclusivamente con la agricultura y la ganadería, por ejemplo.

De hecho, el aprecio y respeto por su comunidad destacó entre los tres resultados positivos más importantes exteriorizados por los estudiantes. Pero, además, el proceso les inculcó el respeto por las 
diferentes creencias y opiniones de los demás, el valor del trabajo colectivo y la interacción con compañeros, así como el interesarse más por rescatar las costumbres y tradiciones que caracterizan la identidad local, tener mejor definidos los criterios sobre lo que significa ruralidad y, por ende, el sentirse más orgullosos de ser upaleños. Se enfatiza la gran valía que tuvo la población migrante en el desarrollo socioeconómico y cultural de la comunidad y, en general, se revela una mayor motivación por parte de los jóvenes en función de apropiarse de espacios participativos, como juventud rural activa que está orgullosa de su identidad y que la defiende en esos espacios donde comúnmente es tomada desde el adultocentrismo.

El proceso educativo instó a los jóvenes a entender qué significa la ruralidad desde una perspectiva propia y luego a tener la posibilidad de compartirla con su familia o algún conocido. También se destaca la importancia de los migrantes en la fundación y el continuo intercambio tanto cultural como económico con los demás pobladores, en torno a la construcción de esa identidad upaleña. Por otra parte, fue trascendental reflexionar sobre lo que significa vivir en una zona rural transfronteriza y sobre algunas de las problemáticas que limitan el desarrollo socioeconómico de sus pobladores.

Igualmente, los estudiantes mencionaron que su participación en la comunidad puede hacerse más efectiva a través de mayor involucramiento en los distintos espacios comunitarios, aunque reconocieron que estos son escasos para los jóvenes. Nombraron, sin embargo, colectivos juveniles como el Comité de la Persona Joven (CPJ), la Red de Jóvenes Migrantes Transfronterizos, la Municipalidad, la Radio Cultural Upala, la Pastoral Juvenil, la Casa de Derechos de las Mujeres, Visión Mundial, el Comité de Deportes, la Asociación de Desarrollo Integral de Upala y el Instituto de Desarrollo Rural, como algunas de las instancias institucionales mediante las cuales poder incidir en las políticas locales de desarrollo.

Finalmente, se les preguntó a los involucrados acerca de algunas recomendaciones sobre el proceso educativo desarrollado, ante lo cual externaron la necesidad de procurar lapsos menos extensos entre los talleres, así como de realizar más talleres que traten la identidad local. 


\section{Conclusiones}

Esta propuesta educativa reconoce que los territorios de frontera han sido históricamente víctimas de una desigualdad estructural, en la cual la educación formal también tiene una cuota de responsabilidad, al no ajustarse a las condiciones del contexto en el que se inscribe. Ante esta situación, es que la educación no formal se posiciona como una alternativa que permite generar el desarrollo de otros conocimientos, los cuales reconozcan la complejidad y la diversidad de esos territorios. Aunado a esto, comprender que las zonas rurales, por ejemplo, están viendo transformadas sus formas de organización productiva tradicional, debido al embate neoliberal (el cual impacta en los estilos de vida de estas poblaciones), es una tarea pendiente, si lo que se quiere es lograr la inclusión de las comunidades en las políticas de desarrollo. Para ello, hace falta reconocer, además, que la diversidad cultural es un aspecto clave para trascender los discursos nacionalistas que invisibilizan la dinámica transfronteriza propia de los espacios en cuestión y, por ende, para asumir el diálogo intercultural como una característica que ha determinado el devenir de la cultura e identidad de sus habitantes.

El trabajo se enmarcó en las iniciativas lideradas por la Red de Jóvenes Migrantes y Transfronterizos de Upala, con la finalidad de aportar a un tema antes no abordado, como era el caso de la identidad transfronteriza. Las entrevistas realizadas arrojaron los insumos necesarios para proponer un planteamiento metodológico que atendiera las necesidades, las condiciones y la disponibilidad de los jóvenes. La información recolectada alimentó una propuesta educativa no formal orientada a reflexionar sobre los conceptos de ruralidad, identidad y frontera, con miras a ampliar la concepción acerca de su territorio y, con ello, a fortalecer el arraigo y el compromiso de los jóvenes con la historia pasada, presente y futura de su comunidad. De manera general, la juventud participó de forma activa y se involucró en las diferentes actividades, como parte de los cinco talleres realizados, manifestando, en la mayoría de los casos, que su percepción acerca de lo que significa habitar un territorio transfronterizo, en relación con su historia y cultura, había contribuido a reforzar sentimientos de aprecio y respeto, motivadores para seguir trabajando por el desarrollo de su comunidad. Todo esto, en una propuesta metodológica que contribuyó a una participación construida sobre la base de la confianza, el aprendizaje 
colaborativo y el juego. Como aporte final y con el fin de que el proceso generado pueda replicarse con otras poblaciones a futuro, se elaboró el Manual de formación para el empoderamiento de liderazgos jóvenes rurales transfronterizos, en el cual se describe una serie de actividades que van desde la reflexión en torno a la identidad personal, pasando por la familiar, hasta la comunitaria, que favorezcan la participación de los jóvenes en la defensa de su identidad y de su derecho a ser partícipes de la toma de decisiones de los gobiernos locales.

El trabajo ha permitido constatar que se requiere mayores esfuerzos orientados a fortalecer la reconstrucción de miradas más amplias sobre lo que significa vivir en una zona transfronteriza. Para ello, es necesario luchar por una educación menos estandarizada, ya sea desde iniciativas formales o de las no formales, que promuevan el empoderamiento de los jóvenes, con miras a defender su derecho a una identidad y un desarrollo local. La participación de la juventud en más espacios es fundamental, por lo que la capacitación en temas de liderazgo, por ejemplo, se torna necesaria para romper con el adultocentrismo que aún predomina en los territorios rurales. Asimismo, el tema del fortalecimiento de la identidad y la cultura locales, al igual que de la memoria histórica como medio para fomentar el diálogo intergeneracional, se proponen como aspectos que precisan aún ser abordados. Por último, surge la demanda de que existan políticas públicas, las cuales incluyan las necesidades de la juventud rural en temas de empleo, educación, cultura, participación política, entre otras, que promuevan el desarrollo diferenciado de las regiones, pero siempre en un marco de equidad relacionado con las zonas urbanas.

En un contexto marcado por la falta de empleos, el poco acceso a la educación y la discriminación hacia las mujeres y la población migrante, la educación se convierte en una herramienta fundamental para insertar los sectores menos favorecidos, como es el caso de los jóvenes. En estos, sin duda, parece recaer la responsabilidad de construir un mejor futuro para la región y por ello este proyecto se enfoca en la juventud como agente transformador de su realidad.

Como adendum a estas conclusiones, debe mencionarse la tragedia acaecida el jueves 24 de noviembre de 2016, a raíz de la llegada del huracán Otto, que dejó diez muertos, cuarenta mil afectados y miles de millones de colones en pérdidas. El cantón de Upala fue el escenario de los peores estragos ocurridos en el país debido a ese huracán y esto 
ha contribuido a incrementar aún más los problemas que los upaleños deben resolver para construir un futuro mejor. A las limitaciones antes mencionadas ahora se le añade la preocupación por su vulnerabilidad, al encontrarse en un territorio plano propenso a las inundaciones por los ríos que lo atraviesan. Si bien las advertencias sobre el riesgo de inundación ya habían sido señaladas por algunos organismos nacionales, sobre todo desde el crecimiento desmedido y poco planificado de la comunidad en los últimos años, no fue sino hasta la llegada de Otto que este demostró la fragilidad del cantón ante este tipo de desastres. Con todo, la catástrofe ha servido, al mismo tiempo, para poner el foco de atención en una comunidad cuyo abandono, a lo largo de los años, quedó patente para todo el país, tras el paso de la catástrofe.

Es notable que, después del paso del huracán Otto, el cantón de Upala no ha escatimado en esfuerzos por salir adelante, en coordinación con diferentes instituciones de gobierno, el sector privado, así como con las diferentes campañas de donación llevadas a cabo por diversas organizaciones y familias que han dado un voto de solidaridad. Según datos del Alfaro (2017), en lo que se refiere a reconstrucción de caminos y puentes, a la fecha existen en ejecución sesenta y cinco proyectos con los que se planea la recuperación total de la infraestructura vial. En cuanto a la construcción de viviendas afectadas, se trabaja en coordinación con instituciones como el BAHNVI y otras entidades financieras. Por su parte, el Instituto Mixto de Ayuda Social (IMAS) ha entregado mobiliario y electrodomésticos a las familias afectadas.

A meses luego de la catástrofe, todavía se sigue trabajando. La comunicación entre el gobierno central y los líderes comunales ha permitido escuchar las necesidades de la comunidad. En este sentido, la experiencia del huracán también se convierte en una oportunidad de desarrollo para una localidad con índices de desarrollo humano entre los más bajos. Ahora, sin embargo, a partir de la inversión en infraestructura y de los proyectos de apoyo a los microempresarios que están ejecutando las diferentes instituciones, tanto de gobierno como del sector privado, se tiene la esperanza de un mejor futuro. En este contexto, los jóvenes enfrentan el reto de poder incidir como nunca antes en la redefinición de un nuevo futuro para su comunidad, lo cual pasa, necesariamente, por el replanteamiento de los elementos identitarios que hasta ese momento han sido la base sobre la que experimentan su edad, en aras de lograr construir más y mejores oportunidades para todos. 


\section{Referencias}

Alfaro, J. (26 de junio, 2017). Reconstrucción de Costa Rica tiene más fuerza que la furia de Otto. Recuperado de http://gobierno.cr/ reconstruccion-de-costa-rica-tiene-mas-fuerza-que-furia-del-huracan-otto/

Angulo, L.; Morera, D. y Torres, N. (2010). El proceso pedagógico de la escuela rural unidocente y multigrado centroamericana. Su evolución, condiciones actuales y perspectivas de desarrollo. San José: El Atabal S. A.

Delgado O. y Córdoba G. (2012). Informe Comunidades Fronterizas: Upala. Ministerio de Vivienda y Asentamientos Humanos. Recuperado de https://exnet.mivah.go.cr/Documentos\%5Cinvestigaciones_diagnosticos\%5Cdiagnosticos_planes_intervencion \%5C2012\%5CUPALA_ALAJUELA\%5CMODELO PLAN_INTERVENCION_UPALA_FRONTERA.pdfDuarte, K. (2006). Discursos de resistencias juveniles en sociedades adultocéntricas. San José: DEI.

MIDEPLAN. (2013). Región Huertar Norte. Plan de Desarrollo 2030. San José: MIDEPLAN.

León-Araya, A. (2015). Desarrollo geográfico desigual en Costa Rica. El Ajuste estructural visto desde la Región Huetar Norte (19852005). San José: Editorial UCR.

Programa Conjunto Juventud, Empleo y Migración. (2011). Encuesta sobre la realidad de las personas jóvenes de Upala. Recuperado de http://www.nacionesunidas.or.cr/dmdocuments/B_Encuesta_Upala.pdf

Rodríguez, F. (2001). Región, identidad y cultura. San José: Ediciones Perro Azul.

Vidal, M.; Torres, N.; Cerdas, Y. y Vargas, M. (2015). Comunidades rurales aprendientes: Nuevas rutas en la educación continua. Una educación continua donde aprendimos a sentir, pensar, vivir, compartir, hacer, decir San José: El Atabal S. A.

Zúñiga, M. (2010). Pensar a las personas jóvenes: Más allá de modelos o monstruos. San José: DEI. 
